\title{
The surgical management of low grade single level adult degenerative spondylolisthesis: posterolateral fusion versus posterior lumbar interbody fusion
}

\begin{abstract}
Posterolateral Fusion (PLF) and Posterior Lumbar Interbody Fusion (PLIF) are procedures that can be undertaken for the surgical management of degenerative low grade spondylolisthesis. Although there is tentative evidence in the literature that PLIF provides an increased fusion rate and therefore an improvement in symptomology, PLIF surgery does carry an increased peri- and post-operative complication rate. The aim of this study was to establish which surgical intervention has the better outcomes.

A prospective cohort study was undertaken of patients who underwent a single level instrumented fusion for low grade degenerative lumbosacral spondylolisthesis between 2008 and 2010. There were 56 patients in total, with 36 patients in the PLF group (group1), and 20 patients in the PLIF group (group 2). Follow-up was at 3 month, 1 year and 3 year post operatively. Visual analogue leg pain score (VAS), Oswestry Disability Index (ODI) for back pain, and SF 12 Health Survey were measured pre and 3 year post operatively. All patients were assessed by MRI scans preoperatively. Standardized lumbosacral radiographs were taken preoperatively, and at 3 month, 1 year and 3 year postoperatively, to assess radiological union. In the PLF group, the VAS score improved from 8 to 2, the SF 12 score from 25 to 76, and the ODI score from 36 to 8. In the PLIF group, the VAS score improved from 8 to 4, the SF 12 score from 27 to 72 , and the ODI score from 38 to 12. All improvements were statistically significant. There was a statistically larger improvement margin in all scores in the PLF group than in the PLIF group. Radiological union was demonstrated in $91 \%$ of all patients, with $95 \%$ of patients in each group demonstrating at least, unilateral lateral mass bone graft incorporation. We conclude that PLF surgery is superior to PLIF surgery for the surgical management of low grade single level degenerative spondylolisthesis.
\end{abstract}

Keywords: Degenerative spondylolisthesis, Posterolateral fusion, Posterior lumbar interbody fusion, Back pain, Leg pain, Decompression surgery
Volume I Issue I - 2014

\author{
Khaldoun El-Abed, MJ Barakat \\ Department of Orthopedics \& Physiotherapy,Al-Zahra Private \\ Hospitals, UAE
}

Correspondence: Khaldoun El-Abed, Department of Orthopedics \& Physiotherapy,Al-Zahra Private Hospitals, Sharjah, P.O.Box 3499, UAE, Tel 0097। 502002I73, Email Khaldoun.elabed@gmail.com

Received: May 05, 2014 | Published: May 12, 2014

\section{Abbreviations}

PLF, Posterolateral Fusion; PLIF, Posterior Lumbar Interbody Fusion; VAS, Visual Analogue Leg Pain Score; ODI, Oswestry Disability Index; RR, Relative Risk; CI, Confidence Interval

\section{Introduction}

Degenerative spondylolisthesis is a common condition which occurs most frequently in the lower lumbar spine, most commonly at the level of L4/L5. The extent of the slip is usually graded using the Meyerding classification with grades I and II representing upto $25 \%$ and $50 \%$ displacement respectively. These are referred to as lowgrade slips. ${ }^{1}$ The initial management of the condition is non-operative. However, should this fail surgical intervention is then considered. ${ }^{2}$

Posterolateral fusion (PLF) has long been the gold standard for the surgical treatment of adult degenerative spondylolisthesis. ${ }^{2,3}$ Posterior Lumbar Interbody Fusion (PLIF) is a relatively novel technique that allows for ' 360 ' degree fusion, using Posterolateral fusion supplemented with the placement of graft-filled cages into the disc cavity after the disc has been removed, using a posterior approach. ${ }^{4}$ Controversy still exists in regards to which technique is superior for the treatment of low grade degenerative spondylolisthesis. ${ }^{5-7}$ The aim of this study is to prospectively evaluate and compare the clinical and radiological results of PLIF and PLF for low grade adult degenerative spondylolisthesis.

\section{Materials and methods}

A prospective cohort study was performed on 56 patients, from 2008 to 2010, who had undergone either PLIF or PLF surgical treatment for adult degenerative low grade spondylolisthesis. Ethical approval was sought and given by the appropriate ethical committee. Thirty six patients were in the PLF group (group1), while 20 patients were in the PLIF group (group 2). All patients had low grade degenerative spondylolisthesis with $71 \%$ at the L4/L5 level. All patients had preoperative unilateral lower limb radiculopathy. All patients had a preoperative MRI scan. All procedures were performed by experienced spinal consultants, without active spondylolisthesis reduction. All procedures involved single level complete bilateral laminectomy to decompress the nerve roots. The corticocancellous bone harvested from the laminae and spinous processes was used as graft material. The harvested bone was cleaned of soft tissue, morcellised and placed over the decorticated transverse processes and inter-transverse 
membrane. Transpedicular Java ${ }^{\circledR}$ Pedicle Screw System (Zimmer, Minneapolis, MN, USA) was used in all cases. The TM-500® Lumbar Interbody Spacer (Zimmer, Minneapolis, MN, USA), made of Trabecular Metal ${ }^{\mathrm{TM}}$ was used for the PLIF cases.

The inclusion criteria included patients with chronic low back pain with or without sciatica, failed conservative therapies of at least 6 month duration prior to surgery, and Grade I degenerative spondylolisthesis with stenotic radicular symptoms and back pain. The exclusion criteria included history of previous spinal surgery, isolated radicular symptoms, cauda equine syndrome, multi-level disease, infections and malignancy. Follow-up was at 3 month, 1 year and 3 year. Clinical assessment was carried out pre-operatively, 3 month post-operatively and 3 year post-operatively. Radiological assessment was carried out pre-operatively, 1 year post-operatively and 3 year post-operatively. Clinical assessment involved the measurement of the 10-point visual analog back pain score (VAS), Oswestry Disability Index (ODI) for back pain, and SF 12 Health Survey. All comorbidities were matched between the patients to reduce error in outcome measurement. ${ }^{8}$

Radiological assessment was in the form of a comparison between standardised antero-posterior and lateral weight-bearing radiographs, to assess radiological bony union. Statistical analysis was carried out using the Wilcoxon matched-pairs signed rank test for each outcome measure at the 3-year mark. For the outcome results, the relative risk (RR) and 95\% confidence interval (CI) were calculated. A chi-square analysis was performed for each outcome to determine the statistical significance. A P-value of $<0.01$ was deemed highly significant, whereas a P-value $<0.05$ was deemed marginally significant. The subjects' variables were described using frequency distribution for categorical variables, and mean and standard deviation for continuous variables. The changes in scores over time were tested using repeated measures analysis.

\section{Results}

There were 56 patients in total; 30 were female and 26 male. The mean age was $52 \pm 6.5$ years (range $42-64$ years). Follow up data Table I The pre-operative, 3 month post-operative and 3 year post-operative clinical parameter outcomes for the PLF group

\begin{tabular}{llll}
\hline PLF Group & Preop & Postop 3 month & Postop 3 year \\
\hline VAS (Back Pain) & $8(6-10)$ & $3(0-5)$ & $2(0-4)$ \\
ODI & $36(30-38)$ & $10(7-14)$ & $8(6-12)$ \\
SF 12 (Version 2) & $25(19-33)$ & $72(61-80)$ & $76(62-82)$ \\
\hline
\end{tabular}

Table 2 The pre-operative, 3 month post-operative and 3 year post-operative clinical parameter outcomes for the PLIF group

\begin{tabular}{llll}
\hline PLIF Group & Preop & Postop 3 month & Postop 3 year \\
\hline VAS (Back Pain) & $8(6-10)$ & $5(3-6)$ & $4(2-5)$ \\
ODI & $38(31-4 I)$ & $16(12-19)$ & $12(9-14)$ \\
SF I2 (Version 2) & $27(18-36)$ & $69(60-75)$ & $72(62-78)$ \\
\hline
\end{tabular}

Citation: El-Abed K, Barakat MJ.The surgical management of low grade single level adult degenerative spondylolisthesis: posterolateral fusion versus posterior lumbar interbody fusion. MOJ Orthop Rheumatol. 20I4; I (I): I-4. DOI: I0.15406/mojor.20I4.01.0000 I 


\section{Results: VAS (Back Pain)}

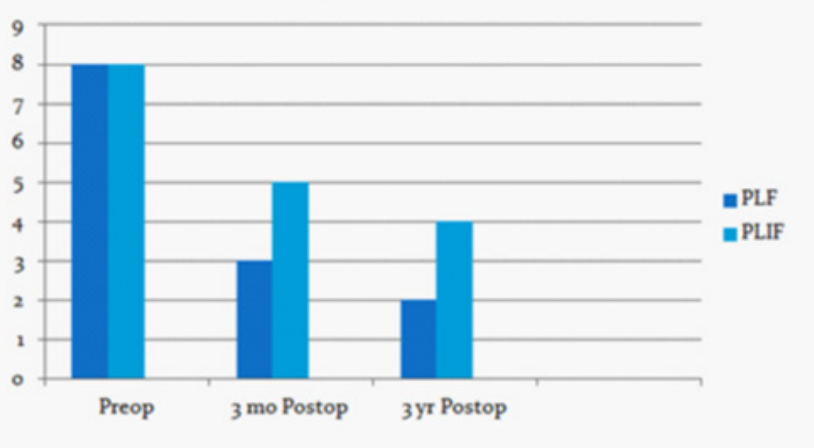

Figure 1 The pre-operative, 3 month post-operative and 3 year post-operative VAS results for PLF vs. PLIF.

\section{Results: ODI}

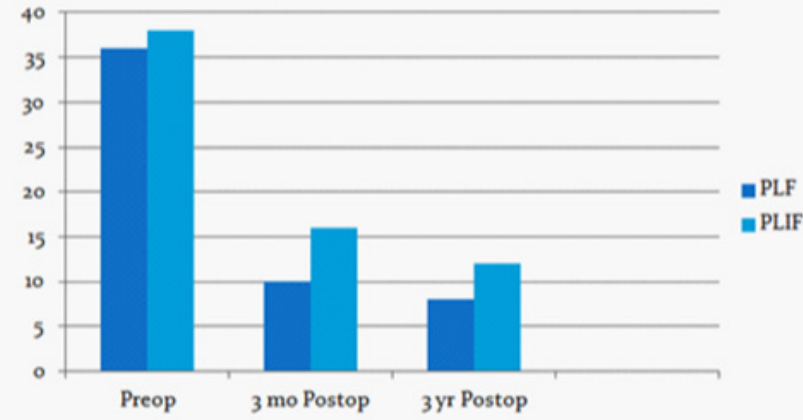

Figure 2 The pre-operative, 3 month post-operative and 3 year post-operative ODI results for PLF vs. PLIF.

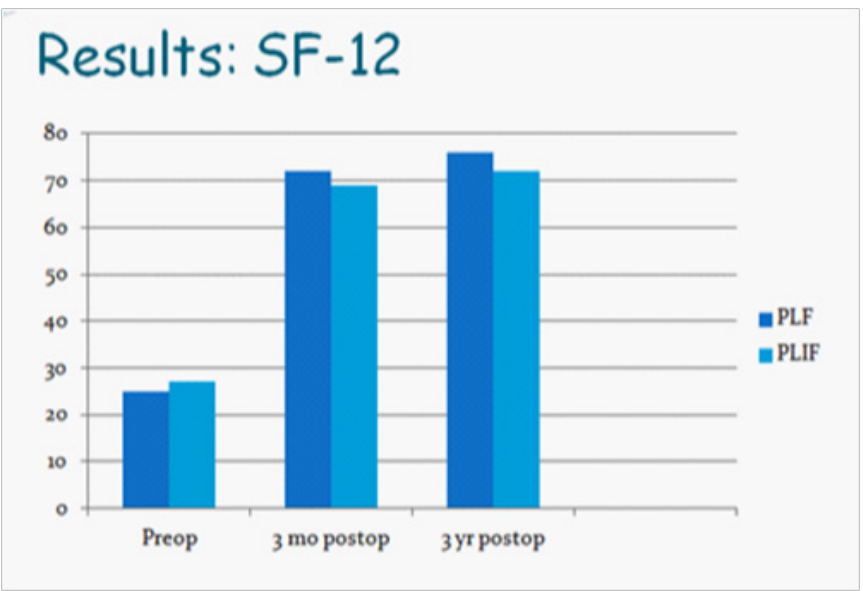

Figure 3 The pre-operative, 3 month post-operative and 3 year post-operative SF-12 results for PLF vs. PLIF.

\section{Discussion}

While posterolateral fusion has been the gold standard for the surgical treatment of low grade degenerative spondylolisthesis, the advantage of anterior column support and fusion in addition to pedicle fixation is the subject of interest and research. ${ }^{1-4}$ Over the last decade, PLIF has become a popular technique for achieving interbody fusion.
The development of the pedicle screw fixation system combined with interbody spacers has been shown to improve the rate of arthrodesis. ${ }^{5-7}$ In addition to that, the use of interbody spacers has shown better results in term of disc height maintenance and in maintaining direct neural decompression, than using bone graft alone. PLIF, however, requires significant retraction on the dural sac and nerve roots. As a result, it is associated with higher risks of cerebrospinal fluid leakage, dysesthetic 
nerve pain syndrome, nerve root injury, epidural fibrosis, as well as cage migration. ${ }^{9-11}$ In our study, multivariate analysis was used to account for potential covariates and identify factors associated with an increased complication risk - the only peri-operative complications occurred in the PLIF group, with 2 cases of dural tears, 2 cases of epidural hematomas, and 2 cases of dysesthetic nerve pain syndrome secondary to genitofemoral nerve injury.

Regarding fusion rate following surgery, there is moderate evidence in the literature that suggests that there is no difference in the fusion rates between PLF and PLIF. There is very sparse evidence suggesting that the functional outcomes are better after anterior column support than other fusion models..$^{5-7}$ In our study, we noted a significant improvement in all functional scores in the PLF group at the 3 month mark following surgery with further marginal improvement at the 3 year post-operative period. This improvement was significantly better than the improvement noted in the PLIF group over the same periods. From a radiological point of view, both groups demonstrated a $90 \%$ radiological union rate. We noted the increased operating time, blood loss and complication rate associated with PLIF surgery.

\section{Conclusion}

Both PLIF and PLF surgery have demonstrated an improvement in functional outcomes at the 3 year post-operative period, with excellent radiological union demonstrated at the 3 year post-operative radiograph. The PLF group has demonstrated a better improvement in functional outcomes, and this coupled with the increased operating time, blood loss and complication rate associated with PLIF surgery, would make us conclude that PLF surgery is the choice for the surgical management of low grade degenerative spondylolisthesis.

\section{Acknowledgments}

None.

\section{Conflicts of interest}

The author declares that there is no conflict of interest.

\section{References}

1. Sengupta DK, Herkowitz HN. Degenerative spondylolisthesis: review of current trends and controversies. Spine. 2005;30(6):71-81.
2. Fritzell $\mathrm{P}$, Hagg $\mathrm{O}$, Wessberg $\mathrm{P}$, et al. Lumbar fusion versus nonsurgical treatment for chronic low back pain: a multicenter randomized controlled trial from the swedish lumbar spine study group. Spine. 2001;26(23):25212532 .

3. Fischgrund JS, Mackay M, Herkowitz HN, et al. Degenerative lumbar spondylolisthesis with spinal stenosis: a prospective, randomized study comparing decompressive laminectomy and arthrodesis with and without spinal instrumentation. Spine. 1997;22(24): 2807-2812.

4. DiPaola CP, Molinari RW. Posterior lumbar interbody fusion. $J$ Am Acad Orthop Surg. 2008;16(3):130-139.

5. Dantas FL, Prandini MN, Ferreira MA. Comparison between posterior lumbar fusion with pedicle screws and posteriorlumbar interbody fusion with pedicle screws in adult spondylolisthesis. Arq Neuropsiquiatr. 2007;65(3B):764-770.

6. Fritzell $\mathrm{P}$, Hagg $\mathrm{O}$, Wessberg $\mathrm{P}$, et al. Chronic low back pain and fusion: a comparison of three surgical techniques: a prospective multicenter randomized study from the swedish lumbar spine group. Spine. 2002;27(11):1131-1141.

7. Lee CS, Hwang CJ, Lee D-H, et al. Fusion rates of instrumented lumbar spinal arthrodesis according to surgical approach: A systemic review of randomized trials. Clin Orthop Surg. 2011;3(1):39-47.

8. Mofidi A, O'Connor D, El-Abed K, et al. Functional outcome study of patients after surgical decompression for lumbar spinal stenosis: effects of concomitant pathology. J Spine Disord Tech. 2002;15(5):377-383.

9. Kimura H, Shikata J, Odate S, et al. Risk factors for cage retropulsion after posterior lumbar interbody fusion: analysis of 1070 cases. Spine. 2012;37(13):1164-1169.

10. Chen L, Yang H, Tang T. Cage migration in spondylolisthesis treated with posterior lumbar interbody fusion using BAK cages. Spine. 2005;30(19):2171-2175.

11. Hosono N, Namekata M, Makino T, et al. Perioperative complications of primary posterior lumbar interbody fusion for nonisthmic spondylolisthesis: analysis of risk factors. J Neurosurg Spine. 2008;9(5):403-407. 